\title{
Mind wandering minimizes mind numbing: Reducing semantic-satiation effects through absorptive lapses of attention
}

\author{
Benjamin W. Mooneyham ${ }^{1}$. Jonathan W. Schooler ${ }^{1}$
}

Published online: 6 January 2016

(C) Psychonomic Society, Inc. 2016

\begin{abstract}
Mind wandering is associated with perceptual decoupling: the disengagement of attention from perception. This decoupling is deleterious to performance in many situations; however, we sought to determine whether it might occur in the service of performance in certain circumstances. In two studies, we examined the role of mind wandering in a test of "semantic satiation," a phenomenon in which the repeated presentation of a word reduces semantic priming for a subsequently presented semantic associate. We posited that the attentional and perceptual decoupling associated with mind wandering would reduce the amount of satiation in the semantic representations of repeatedly presented words, thus leading to a reduced semantic-satiation effect. Our results supported this hypothesis: Self-reported mind-wandering episodes (Study 1) and behavioral indices of decoupled attention (Study 2) were both predictive of maintained semantic priming in situations predicted to induce semantic satiation. Additionally, our results suggest that moderate inattention to repetitive stimuli is not sufficient to enable "dishabituation": the refreshment of cognitive performance that results from diverting attention away from the task at hand. Rather, full decoupling is necessary to reap the benefits of mind wandering and to minimize mind numbing.
\end{abstract}

Keywords Semantic priming $\cdot$ Repetition effects $\cdot$ Mind wandering $\cdot$ Sustained attention

Benjamin W. Mooneyham

bwmooneyham@gmail.com

1 Department of Psychological \& Brain Sciences, University of California, Santa Barbara, Santa Barbara, CA, USA

\section{Introduction}

Think of a word, any word; let it be as meaningful to you as possible. Now mentally repeat that word in your head over and over again. If you are like most people, you will soon notice an astonishing phenomenon: The word quickly loses all meaning. The continuous encountering of a word can produce a remarkably mind-numbing effect, referred to as "semantic satiation" (Balota \& Black, 1997; Smith \& Klein, 1990). Such a dramatic effect of repetition, and mental habituation toward a stimulus, leads to an important question: Have our brains evolved any processes to counteract the mind numbing that can accompany repetition? Here we suggest one such antidote: mind wandering, the decoupling of attention from external stimuli toward thoughts unrelated to the current task.

The recent explosion in interest in the phenomenon of mind wandering has produced a substantial array of findings that implicate it as a detrimental process. Performance in a host of domains, including reading comprehension (e.g., Franklin, Smallwood, \& Schooler, 2011), sustained attention (e.g., Cheyne, Solman, Carriere, \& Smilek, 2009), and working memory capacity (Mrazek, Smallwood, Franklin, et al., 2012b), has been examined in relation to mind wandering, and in each case the impact of mind wandering has been notably negative. However, mind wandering is such a prevalent occurrence in the lives of most individuals that it seems unlikely that it could be purely maladaptive (Mooneyham \& Schooler, 2013). Although most research on the impact of mind wandering has been focused on the negative consequences, recent research has begun to implicate its potential benefits in domains such as planning (Baird, Smallwood, \& Schooler, 2011) and creativity (Baird et al., 2012; Zedelius \& Schooler, 2015). Here we consider another possible benefit: namely, that mind wandering may provide a brief break that 
attenuates habituation. The dishabituation hypothesis predicts that the alternation of focus between external stimuli and mind-wandering content may allow for "refreshed" processing of the external stimuli, thereby minimizing the "mindnumbing" effects of habituation. In the following two studies, we investigated this hypothesis by examining whether mind wandering can minimize semantic satiation, leading to greater semantic-priming effects for repetitively presented verbal stimuli.

\section{Semantic priming and semantic satiation}

Semantic priming is an automatic process in which the perception of a word initiates the activation of a network of semantically related lexical associates (e.g., Balota \& Black, 1997). This effect is typically measured through speeded reaction times (RTs) to words semantically related to a previously presented target word. Priming is a fickle process, however, since simply increasing the exposure of the prime can reduce its priming effectiveness. Such is the case in the phenomenon of "semantic satiation" (Balota \& Black, 1997; Smith \& Klein, 1990), which describes the finding that, although a briefly presented word can prime responses to a subsequently presented related word, presenting the prime word repeatedly can diminish this priming effect. Essentially, then, semantic satiation represents an ironic process in which the repeated presentation of potentially useful or relevant information can actually hinder one's ability to use that information readily.

\section{Mind wandering and perceptual decoupling}

One of the hallmark characteristics of mind wandering is that it is associated with perceptual decoupling (Schooler et al., 2011; Smallwood, Beach, Schooler, \& Handy, 2008; Smallwood et al., 2011). Perceptual decoupling describes the process by which our behaviors and cognitions become less constrained by the perceptual information surrounding us while mind wandering. For instance, when mind wandering while reading, individuals' eye movements are less sensitive to the lexical features of the text (such as word length and frequency; e.g., Reichle, Reineberg, \& Schooler, 2010). This reduced sensitivity to the informational characteristics of the external environment during mind wandering has further been shown to be associated with a concomitant reduction in the physiological responses to external stimuli (Franklin, Broadway, Mrazek, Smallwood, \& Schooler, 2013; Schooler et al., 2011; Smallwood et al., 2008; Smallwood et al., 2011).

If our brains become largely decoupled from external input while mind wandering, then mind wandering should also provide an antidote to habituation in situations in which identical stimuli are presented repeatedly, a prime example of which is the context in which semantic satiation occurs.

\section{Study 1}

We sought to explore the possibility that mind wandering may reduce semantic satiation effects, by embedding experiencesampling probes within a standard paradigm for assessing semantic satiation. Participants made "related"/"unrelated" judgments about word pairs that varied with respect to the number of times that the first word in each pair was presented before the second word appeared. "Long" trials included numerous repetitions of the first word, thereby jeopardizing semantic priming and encouraging semantic satiation. In contrast, "short" trials included only a few repetitions of the first word, thereby (hypothetically) preserving priming. Throughout the task, participants received experience-sampling probes to characterize their performance on the basis of whether or not they had mind wandered. We hypothesized that a semantic-satiation effect would be observed for trials for which participants reported being on task, but that it would be reduced on trials that participants characterized as including mind wandering. In other words, we predicted that when participants mind wandered, semantic priming would be preserved even after many presentations of the first word in a given word pair.

\section{Method}

Participants A total of 116 (65 females, 51 males) undergraduate students participated in exchange for course credit (mean age $=19.13$ years, $S D=.86$ ). This study was approved by the University of California Santa Barbara's Institutional Review Board, and informed consent was obtained from each participant at the beginning of the experimental session.

Materials In all, 30 pairs of related words and 30 pairs of unrelated words were used, taken from Wible and colleagues' (2006) study of semantic priming. Related word pairs had high semantic connectivity, whereas unrelated word pairs had low semantic connectivity. The words in both the related and unrelated conditions were matched for frequency, number of syllables, and familiarity.

Procedure Participants completed 60 trials of the following procedure: For each trial, a single noun was presented centrally onscreen for 1,500 ms, and then removed for $500 \mathrm{~ms}$. This process repeated until the first noun had been presented two, three, or four times (the "short" trials) or 28, 29, or 30 times (the "long" trials); the number of first-noun repetitions for each trial was determined pseudorandomly, such that 30 trials had a short duration and 30 trials had a long duration. After the repetitive presentation of the first noun, a second noun was presented once, and participants responded with a keypress whether they thought the two nouns presented in the trial were semantically related. The related and unrelated word pair trials were distributed randomly throughout the task. Participants were instructed 
to make their responses "as quickly and accurately as possible" and as soon as they observed the second word in the pair; the second word remained onscreen until a response was made. Participants responded by keypress and received auditory feedback for correct and incorrect responses.

In between trials, participants were presented with the following mind-wandering thought probe onscreen: "Did your mind wander during the previous trial?" Participants indicated their response by keypress.

\section{Results}

For the analysis, participants' responses were coded as either correct or incorrect, and binned into categories of "on task" or "mind wandering" based on the thought-probe responses. Furthermore, each trial could be categorized as either "short" or "long" and as featuring either a related or an unrelated noun pair. In order to exclude outliers, trials on which participants' RTs were less than $200 \mathrm{~ms}$ or greater than $3 \mathrm{~s}$ were discarded, as were trials on which a participant's RT was more than 2.5 standard deviations beyond their mean RT during the task.

Although our principal hypothesis does not make a prediction regarding the effect of mind wandering on accuracy within this task, we sought to determine whether mind wandering led to any global differences in performance. To do this, we calculated each participant's accuracy separately for trials on which he or she was either "on task" or "mind wandering." Employing a paired $t$ test, we found that accuracy was significantly lower on average for trials on which participants reported mind wandering $(M=.83, S D=.22)$ than for on-task trials $(M=.98, S D=.04): t(113)=-7.44, p<.001$. A likely reason for the detrimental effect of mind wandering on accuracy observed here is that individuals may have inadequately encoded the first of the paired words during some short trials as a result of mind wandering, reflecting poor attentional focus on the task itself and producing errors on this relatively easy task. To assess this possibility, we compared participants' accuracy as a function of trial duration and thought-probe response using a 2 (Duration: long or short) $\times 2$ (Thought-Probe Response: on task or mind wandering) repeated measures analysis of variance (ANOVA). This ANOVA revealed main effects of both duration $[F(1,95)=25.22, p<.001]$ and thought-probe response $[F(1,95)=72.83, p<.001]$, as well as a significant Duration $\times$ Thought-Probe Response interaction $[F(1,95)=18.72, p<.001]$. Participants' mean accuracies across these four conditions are reported in Table 1. Participants performed less accurately on short-duration trials overall, and also less accurately when they reported mind wandering. Moreover, as indicated by the interaction effect, the effect of mind wandering on accuracy was most detrimental among the short-duration trials, suggesting that the negative impact of mind wandering on accuracy within this task was largely driven by a failure to adequately attend to the taskrelated information at the onset of individual trials.

To demonstrate the effect of mind wandering on the semantic-satiation effect by directly comparing "on-task" and "off-task" trials, we calculated the mean difference in the RTs for related and unrelated word pairs; this measure reflects the priming effect, and was calculated separately for "on-task" and "off-task" trials at each duration condition. Using the priming effect as the dependent variable, a 2 (Duration: short or long) $\times 2$ (Thought-Probe Response: on task or mind wandering) repeated measures ANOVA yielded a main effect of duration $\left[F(1,51)=6.04, p<.05, \eta^{2}=.106\right]$, such that participants generally showed the greatest priming at the short durations, whereas the main effect of thought-probe response was nonsignificant $\left[F(1,51)=3.24\right.$, n.s., $\left.\eta^{2}=.061\right]$. Most importantly, the Duration $\times$ Thought-Probe Response interaction was significant $\left[F(1,51)=13.36, p=.001, \eta^{2}=\right.$ .208]. Although both "on-task" and "mind-wandering" trials were associated with semantic priming within the short trial durations, priming only occurred within the long trial durations when "mind wandering" was reported (see Fig. 1). A paired $t$ test comparing the priming effects for "on-task" versus "mind-wandering" long-duration trials showed a significant effect: $t(73)=-6.53, p<.001$.

\section{Study 2}

Study 1 provided evidence that mind wandering can encourage dishabituation, and thereby attenuate the mind-numbing effects of repetition. However, Study 1 possessed several limitations. First, it relied on self-reports of mind wandering that may have been influenced by participants' performance on the semantic judgment task, given that these reports were made

Table 1 Study 1: mean accuracy (proportion of trials responded to correctly) by trial length (i.e., number of repetitions) and task focus (on task vs. mind wandering)

\begin{tabular}{lllll}
\hline Trial Length (number of repetitions): & Short & \multicolumn{2}{l}{ Long } \\
\hline Task Focus: & On Task & Mind Wandering & On Task & Mind Wandering \\
& Mean $(S D)$ & Mean $(S D)$ & Mean $(S D)$ & Mean $(S D)$ \\
Mean accuracy & $.976(.048)$ & $.752(.309)$ & $.987(.039)$ & $.895(.160)$ \\
\hline
\end{tabular}

Standard deviations are reported in parentheses. 


\section{Priming Effects by Trial Duration and Task Focus}

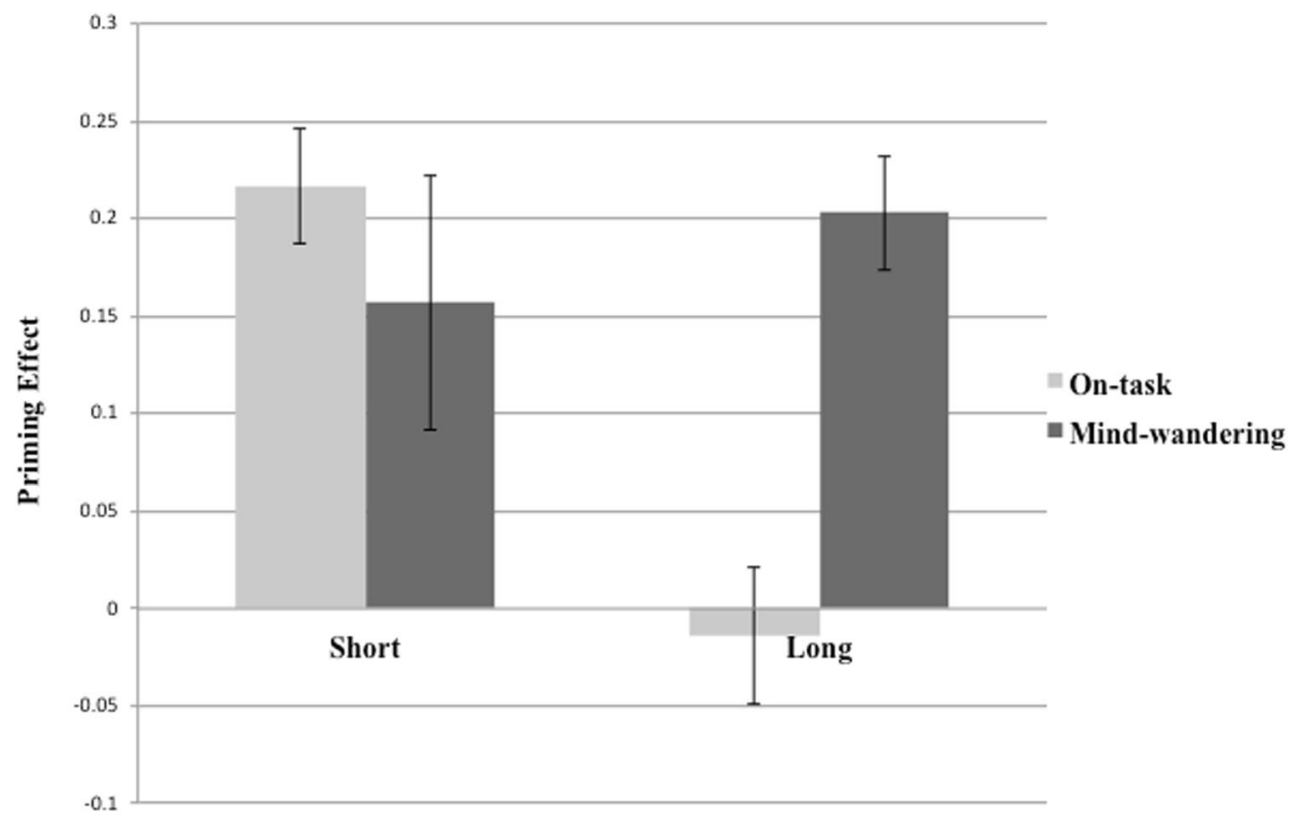

Fig. 1 Study 1: priming effects for short- versus long-duration trials, displayed by task focus (on task vs. mind wandering). Priming effect = Average RT for unrelated word pairs - Average RT for related word pairs. Error bars represent standard errors

immediately after making the semantic judgments. It is therefore possible that semantic judgment accuracy could have been used heuristically in participants' mind-wandering selfassessments. Second, the retrospective nature of the mindwandering reports may also have reduced the accuracy with which participants judged their mental state during each trial. Third, Study 1 provided only a dichotomous measure of mind wandering, which does not allow for assessments of the possible influence of the degree of task disengagement within semantic-satiation trials.

We sought to validate the findings from Study 1 within a paradigm that addressed these possible concerns. To do so, we developed a new test of semantic satiation, embedded within a "sustained attention to response task" (SART) paradigm (Robertson, Manly, Andrade, Baddeley, \& Yiend, 1997). The SART is a go/no-go task commonly used to assess individuals' propensities to exhibit lapses in attention (e.g., Cheyne et al., 2009). The standard SART procedure involves

Table 2 Study 2: multiple regression predicting semantic judgment accuracy across the entire task using SART errors and omissions

\begin{tabular}{lllc}
\hline $\begin{array}{l}\text { Dependent Variable: } \\
\text { Semantic-judgment } \\
\text { accuracy (entire task) }\end{array}$ & $\beta$ & $t$ & $p$ \\
\hline Proportion of SART errors & -.464 & -3.18 & .004 \\
Proportion of omission errors & -.526 & -3.54 & .001 \\
& $R^{2}=.42, F(2,27)=9.65, p<.001$ \\
\hline
\end{tabular}

responding to numbers. In the newly devised "semantic-satiation SART" version of this paradigm, we modified the procedure so that the stimuli were words. This enabled us to observe semantic satiation within the context of a paradigm that offered behavioral markers of mind wandering.

Notably, the SART produces several behavioral indices that have been associated with different degrees of mind wandering (Mrazek, Smallwood, \& Schooler, 2012a). Two types of errors occur within the SART: errors of commission (failures to withhold a response to "no-go" stimuli; known as "SART errors") and errors of omission (failures to respond to "go" stimuli). These errors reflect differential degrees of task disengagement. Errors of commission are largely driven by the speeding of RTs and are indicative of fluctuations in the exertion of attentional control on the task demands, whereas errors of omission represent a stronger decoupling from the task environment and can be considered to reflect more pronounced degrees of mind wandering (Cheyne et al., 2009). In short, behavioral indices from the newly devised semantic-satiation SART provide an avenue through which to address the main limitations of Study 1, since they are not obtained by self-report, are nonretrospective, and allow for examinations of the effects of varying degrees of inattention.

\section{Method}

Participants Thirty (16 females, 14 males) undergraduate students participated in exchange for course credit (mean 
Table 3 Study 2: predicting semantic judgment accuracy and semantic priming within long trials by the proportion of SART errors and omissions

\begin{tabular}{lll}
\hline $\begin{array}{l}\text { Dependent Variable } \\
\text { (long trials): }\end{array}$ & $\begin{array}{l}\text { Semantic-Judgment } \\
\text { Accuracy }\end{array}$ & $\begin{array}{l}\text { Semantic-Priming } \\
\text { Effect }\end{array}$ \\
\hline Proportion of SART errors & $-.431^{*}$ & -.043 \\
Proportion of omission errors & -.273 & $.398^{*}$ \\
\hline
\end{tabular}

Statistics reported are Pearson product-moment correlations $(r) .{ }^{*} p<.05$.

age $=19.37$ years, $S D=1.35$ ). This study was approved by the University of California Santa Barbara's Institutional Review Board, and informed consent was obtained from each participant at the beginning of the experimental session.

Procedure Participants performed 60 trials of an adapted semantic-satiation SART in which they were to respond via keypress to each presentation of a particular word on screen (go stimulus), but to withhold responses to infrequent stimuli that consisted of random five-character strings of consonants (e.g., "ZHGVT"). No-go targets appeared randomly throughout each trial on $20 \%$ of stimulus presentations. SART trials varied between one and 30 stimulus presentations (not including the nonword no-go stimuli). At the end of each SART trial, a new word was presented on screen (in blue), and participants were asked to determine whether the new word was semantically related to the word that had been presented during the SART portion of the trial. Trials of each repetition value (130) were each presented twice during the experiment, in random order. Word pairs for these trials were identical to those used in Study 1, such that 30 trials featured semantically related word pairs and 30 trials featured unrelated word pairs. Word pair relatedness was counterbalanced throughout the task, such that related and unrelated words pairs occurred equally often across trial lengths.

Table 4 Summary statistics for measures of mind-wandering and semantic judgment accuracy for short versus long trials in Studies 1 and 2

\begin{tabular}{lll}
\hline $\begin{array}{l}\text { Trial Length } \\
\text { (number of repetitions): }\end{array}$ & $\begin{array}{l}\text { Short } \\
\text { Mean }(S D)\end{array}$ & $\begin{array}{l}\text { Long } \\
\text { Mean }(S D)\end{array}$ \\
\hline Study 1 & & \\
$\quad$ Proportion of trials with mind wandering & $.221(.243)$ & $.535(.340)$ \\
$\quad$ Mean semantic-judgment accuracy & $.938(.067)$ & $.956(.054)$ \\
Study 2 & & \\
$\quad$ Proportions of SART errors & $.228(.203)$ & $.212(.129)$ \\
$\quad$ Proportion of errors of omission & $.020(.021)$ & $.012(.022)$ \\
$\quad$ Mean semantic-judgment accuracy & $.880(.109)$ & $.955(.065)$ \\
\hline
\end{tabular}

In Study 1, short trials featured two, three, or four stimulus repetitions, whereas long trials featured 28, 29, or 30 repetitions. In Study 2, short trials featured one to ten stimulus repetitions, whereas long trials featured 21-30 repetitions.

\section{Results}

We sought to examine the contributions of varying levels of disengagement, indexed separately by SART errors of omission and commission, toward semantic judgment accuracy and semantic priming. In Study 1, mind-wandering reports were associated with poorer semantic judgment performance. A similar effect was observed within the SART-based paradigm, in which both the proportion of SART errors made during the task (i.e., the number of no-go target responses divided by the number of no-go targets) and the proportion of omission errors (i.e., the number of go targets that were not responded to divided by the total number of go targets) were negatively associated with semantic judgment accuracy throughout the entire task. Multiple regression analysis indicated unique contributions of each type of error toward semantic judgment accuracy overall (Table 2).

Study 1 also revealed that mind-wandering reports were associated with greater semantic-priming effects for longduration trials. We assessed the relationship between SART errors and omissions and the semantic-priming effect for the one-third of trials with the largest numbers of stimulus repetitions (i.e., 21-30 repetitions). The proportion of SART errors within these long trials did not predict maintained semantic priming on high-repetition trials, whereas the proportion of errors of omission within long trials was positively associated with greater semantic priming (Table 3). Notably, an opposite pattern was observed in relation to semantic judgment accuracy within the longer trials: The proportion of SART errors showed a significant negative association with semantic judgment accuracy, whereas errors of omission did not (Table 3). ${ }^{1}$

\section{General discussion}

These studies collectively demonstrate that mind wandering can eliminate the semantic-satiation effect. In Study 1, we directly assessed mind wandering using a self-report measure and found that whereas the semantic-satiation effect was observed across long trials on which participants reported being on task, no such effect was observed within long "mindwandering" trials. Instead, we found a maintained semanticpriming effect within long-duration "mind-wandering" trials. However, the mind-wandering reports in Study 1 may not

\footnotetext{
${ }^{1}$ The use of SART errors as indicators of inattention may be susceptible to confounding effects of speed-accuracy trade-offs (SATOs; Seli, Jonker, Cheyne, \& Smilek, 2013). One method to statistically control for SATOs is to use the SART RT as a covariate with SART errors in correlational analyses. Although not reported here, we performed each of the present analyses involving SART errors with SART RT included as a covariate. The statistical significance of the relationships between SART errors, semantic judgment accuracy, and semantic priming was unaffected by the inclusion of this covariate.
} 
have been entirely accurate. The mind-wandering measure was self-reported after each semantic judgment, and therefore may have been influenced by judgment accuracy, so that poor performance may have served as a heuristic for self-reporting mind wandering. Furthermore, the retrospective nature of the reports in Study 1 may have reduced participants' ability to accurately assess their mental states within each trial. Finally, it is also possible that the predictable structure of trials in Study 1 may have allowed for strategically timed mind wandering. We therefore implemented a semantic-satiation task within a novel SART task in Study 2, allowing us to assess differing levels of mind wandering separately and indirectly using well-established behavioral indices of mind wandering within the SART. In support of the conclusions of Study 1, we found that errors of omission within longer-duration SART trials actually predicted improved semantic priming within those trials.

The results of Study 2 shed additional insight into the likely mechanism by which mind wandering may reduce semantic satiation. In Study 2, SART errors on long trials did not predict semantic priming, whereas errors of omission did. SART errors are the product of fluctuations in attentional control over the responses demanded by the task, and they are predicted by other indirect measures of attentional fluctuation within the SART (e.g., SART RT, RT coefficient of variation), but in our study their effects were distinguishable from the effects of errors of omission: We did not observe a significant relationship between the proportions of SART errors and errors of omission at either the whole-task or the longer-trial level. Omission errors are thought to reflect the process of decoupling from the task environment, and thus are also the most direct indications of elaborative mind wandering within sustained attention tasks (Cheyne et al., 2009). This decoupling during mind wandering appears to be crucial for the reduction of the semantic-satiation effect.

Decoupling reduces the processing of external stimuli. The dampened processing of the repeatedly presented words during intermittent periods of mind wandering within both experiments may therefore have minimized habituation at either the sensory or the semantic level. Although it seems highly probable that perceptual decoupling underpins mind wandering's capacity to enable maintained semantic priming despite repeated presentations of a semantic prime, the present studies did not include a direct measure of externally directed attention. The present results strongly suggest, however, that future research would reveal evidence that the reduction in semantic satiation observed in this paradigm is associated with a concomitant attenuation in attentional resources directed toward the primes.

Although mind wandering enabled the maintenance of semantic-priming effect for long-duration trials within both experiments, this occurred at a cost in overall accuracy. The negative impact of mind wandering on performance on these tasks was largely confined, however, to trials of shorter durations. Although both studies revealed an overall negative association between mind wandering and semantic-judgment accuracy, neither study demonstrated such a negative relationship when the analyses were confined to longer trials (Table 4); although SART errors did predict negative performance in Study 2, omissions, which reflect more pronounced and decoupled mind-wandering episodes, did not. We hypothesize that the negative effect of mind wandering on accuracy for shorter trials reflects inadequate initial encoding of the prime stimulus prior to the semantic judgments, as this negative effect did not occur during trials in which the prime stimulus was repeated many times.

The results of these studies indicate that mind wandering may indeed play the role of a "dishabituator" under repetitive conditions. Moreover, these data point to the critical importance of full decoupling, or disengagement, to reaping the benefits of refreshed processing of external events. Modest attentional fluctuations, indicative of divided attention, did not produce priming effects under repetitive situations; in fact, these fluctuations were most associated with poorer performance on our semantic-judgment task. In Study 2, full withdrawal from task-oriented responding was required, if only for a moment, to produce refreshed processing of the stimuli and enable semantic priming. Such decoupling from the task demands was typically very infrequent and brief (approximately $2 \%$ of stimulus presentations overall, in Study 2), yet the benefits were measureable.

More generally, these findings suggest that mind wandering may offer "mental breaks" that ameliorate the fatigue of repetitive situations. Ironically, although primary-task performance may be especially compromised by more pronounced episodes of perceptual decoupling, these particularly absorbing lapses may offer the greatest opportunity for dishabituation. When repetition numbs the mind, an immersive mental departure from the here and now may help restore it.

Author note J.W.S. is supported through the Institute of Education Sciences, Grant No. R305A110277 awarded to J.W.S. B.W.M. is supported by a National Science Foundation Graduate Research Fellowship under Grant No. DGE-1144085. The content of this article does not necessarily reflect the position or policy of the U.S. government, and no official endorsement should be inferred.

\section{References}

Baird, B., Smallwood, J., Mrazek, M. D., Kam, J. W., Franklin, M. S., \& Schooler, J. W. (2012). Inspired by distraction: Mind wandering facilitates creative incubation. Psychological Science, 23, 11171122. doi: $10.1177 / 0956797612446024$

Baird, B., Smallwood, J., \& Schooler, J. W. (2011). Back to the future: Autobiographical planning and the functionality of mind-wandering. Consciousness and Cognition, 20, 1604-1611. 
Balota, D. A., \& Black, S. (1997). Semantic satiation in healthy young and older adults. Memory \& Cognition, 25, 190-202.

Cheyne, J. A., Solman, G. J., Carriere, J. S., \& Smilek, D. (2009). Anatomy of an error: A bidirectional state model of task engagement/disengagement and attention-related errors. Cognition, 111, 98-113. doi:10.1016/j.cognition.2008.12.009

Franklin, M. S., Broadway, J. M., Mrazek, M. D., Smallwood, J., \& Schooler, J. W. (2013). Window to the wandering mind: Pupillometry of spontaneous thought while reading. Quarterly Journal of Experimental Psychology, 66, 2289-2294.

Franklin, M. S., Smallwood, J., \& Schooler, J. W. (2011). Catching the mind in flight: Using behavioral indices to detect mindless reading in real time. Psychonomic Bulletin \& Review, 18, 992-997. doi:10. 3758/s13423-011-0109-6

Mooneyham, B. W., \& Schooler, J. W. (2013). The costs and benefits of mind-wandering: A review. Canadian Journal of Experimental Psychology, 67, 11-18. doi:10.1037/a0031569

Mrazek, M. D., Smallwood, J., \& Schooler, J. W. (2012a). Mindfulness and mind-wandering: Finding convergence through opposing constructs. Emotion, 12, 442-448. doi:10.1037/a0026678

Mrazek, M. D., Smallwood, J., Franklin, M. S., Chin, J. M., Baird, B., \& Schooler, J. W. (2012b). The role of mind-wandering in measurements of general aptitude. Journal of Experimental Psychology: General, 141, 788-798. doi:10.1037/a0027968

Reichle, E. D., Reineberg, A. E., \& Schooler, J. W. (2010). Eye movements during mindless reading. Psychological Science, 21, 1300-1310.

Robertson, I. H., Manly, T., Andrade, J., Baddeley, B. T., \& Yiend, J. (1997). "Oops!": Performance correlates of everyday attentional failures in traumatic brain injured and normal subjects. Neuropsychologia, 35, 747-758. doi:10.1016/S0028-3932(97)00015-8

Schooler, J. W., Smallwood, J., Christoff, K., Handy, T. C., Reichle, E. D., \& Sayette, M. A. (2011). Meta-awareness, perceptual decoupling and the wandering mind. Trends in Cognitive Sciences, 15, 319326. doi:10.1016/j.tics.2011.05.006

Seli, P., Jonker, T. R., Cheyne, J. A., \& Smilek, D. (2013). Enhancing SART validity by statistically controlling speedaccuracy trade-offs. Frontiers in Psychology, 4, 265. doi:10.3389/ fpsyg.2013.00265

Smallwood, J., Beach, E., Schooler, J. W., \& Handy, T. C. (2008). Going AWOL in the brain: Mind wandering reduces cortical analysis of external events. Journal of Cognitive Neuroscience, 20, 458-469.

Smallwood, J., Brown, K. S., Tipper, C., Giesbrecht, B., Franklin, M. S., Mrazek, M. D., . . . Schooler, J. W. (2011). Pupillometric evidence for the decoupling of attention from perceptual input during offline thought. PLoS ONE, 6, e18298. doi:10.1371/journal.pone.0018298

Smith, L., \& Klein, R. (1990). Evidence for semantic satiation: Repeating a category slows subsequent semantic processing. Journal of Experimental Psychology: Learning, Memory, and Cognition, 16, 852-861. doi:10.1037/0278-7393.16.5.852

Wible, C. G., Han, S. D., Spencer, M. H., Kubicki, M., Niznikiewicz, M. H., Jolesz, F. A., . . . Nestor, P. (2006). Connectivity among semantic associates: An fMRI study of semantic priming. Brain and Language, 97, 294-305.

Zedelius, C. M., \& Schooler, J. W. (2015). Mind wandering "Ahas" versus mindful reasoning: Alternative routes to creative solutions. Frontiers in Psychology, 6, 834. doi:10.3389/fpsyg.2015.00834 